\title{
Effect of infiltrating time on interfacial reaction and properties of tungsten particles reinforced Zr-based bulk metallic glass composites
}

\author{
*Qing-chun Xiang, Zhi-bo Feng, Wei Zhang, Hong-ding Wang, Ying-lei Ren, Ke-qiang Qiu \\ School of Materials Science and Engineering, Shenyang University of Technology, Shenyang 110870, China
}

\begin{abstract}
The tungsten particles reinforced $\mathrm{Zr}_{41.2} \mathrm{Ti}_{13.8} \mathrm{Cu}_{12.5} \mathrm{Ni}_{10} \mathrm{Be}_{22.5}$ (Vit 1 alloy) bulk metallic glass composites (BMGCs) were prepared by the melt infiltrating casting method with the infiltrating time of 1,5 and 10 min, respectively. The changes of interfacial reaction and compression properties of the bulk metallic glass composites with different infiltrating times were studied. Results show that with the increase of infiltrating time, tiny nanocrystals are generated at the interfacial boundary of tungsten particles and the amorphous matrix, and the size of tiny crystals increases with the infiltrating time. When the infiltrating time is 10 min, polygonal crystals with a larger size are also generated within the amorphous matrix. The compressive strength of the composites also increases with the infiltrating time. When the infiltrating time is $10 \mathrm{~min}$, the compressive strength of the composite reaches $2,030 \mathrm{MPa}$ and the compression strain is $44 \%$. The fracture morphology of the composite materials is in a vein-like pattern and the melting phenomenon is found on the fracture surface. In addition, the density of the shear bands during the compressive tests of the composite materials increases with the infiltrating time.
\end{abstract}

Key words: Zr-based metallic glass; infiltrating time; interfacial reaction; shear bands; compressive strength CLC numbers: TG146.4 ${ }^{+} 14 \quad$ Document code: A

Article ID: 1672-6421(2020)04-253-07

$\mathrm{B}$ ulk metallic glasses (BMGs) have many potential applications due to their unique properties, such as superior strength, high hardness, excellent corrosion resistance and high wear resistance ${ }^{[1]}$. In general, the plastic deformation of metallic glasses is localized in the narrow shear bands, followed by the rapid propagation of these shear bands and the catastrophic fracture without plasticity ${ }^{[2]}$.

Many efforts have been focused on improving the ductility of BMGs by fabrication of BMG matrix composites $^{[3-6]}$. To date, the bulk metallic glass composites (BMGCs) containing in-situ formed or/and ex-situ added crystalline phases have been developed ${ }^{[7,8]}$. The two categories of BMGCs exhibit enhanced plasticity due to the generation of multiple shear bands in the deformation process.

In the ex-situ phase reinforced BMGCs, the second phase preform is placed in a mold, and subsequently, the melt is infiltrated into the preform. Herein, among

\section{*Qing-chun Xiang}

Male, born in 1972, Professor. His research interests mainly focus on metal solidification, new cast alloys, and casting process simulation.

E-mail: xiangqchun@yeah.net

Received: 2020-05-06; Accepted: 2020-06-19 the ex-situ added crystalline phases, fibers and particles are the most widely used reinforcements. Composites reinforced with fibers may show an anisotropic feature, while composites reinforced with particles usually display the isotropic characteristic.

Due to the unique properties of particles reinforced BMGCs, the deformation and fracture behavior of these materials have been widely investigated. For example, the Ta particles reinforced amorphous matrix composite has been extensively studied for its excellent plasticity ${ }^{[9]}$. In addition, tungsten materials (fibers or particles) have been extensively adopted as the reinforcements in the composites due to their good comprehensive properties and good wettability in the amorphous matrices ${ }^{[10,11]}$. In general, the introduction of the particles reinforcing phases not only affects the mechanical properties of the composites, but also changes their fracture process ${ }^{[12]}$.

The infiltrating casting method is one of the main methods for preparing the particles reinforced composite materials. Due to the nature of the particles, the composite material is usually accompanied by an interfacial reaction, and the influence of the preparation conditions on the interfacial reaction is also the main research object in this field. However, there is not much research on 
the interfacial reaction of the particles reinforced amorphous composites. Most of the researchers are mainly concentrated on the fiber-reinforced amorphous composites, such as the tungsten fiber or $\mathrm{SiC}$ fiber-reinforced composites ${ }^{[13-15]}$.

Few reports can be found about the relationships among the process parameters, interface characteristics, macroscopic mechanical properties and fracture mechanism of the tungsten particles reinforced Zr-based amorphous composites. In fact, since the infiltrating temperature is higher than the melting point of the Zr-based amorphous alloy during the preparation of the composite materials, different degrees of reaction are prone to occur at the interface between the reinforcement phase and the matrix, such as dissolution, diffusion and element segregation. Undoubtedly, the degree of interfacial reaction is closely related to the types of the matrix and the reinforcement phase, as well as the preparation process parameters. Since the structure and properties of the interfaces play a decisive role in the performance of the composite materials, it is very necessary to study the relationship between the preparation process parameters of the amorphous composites and the degree of interfacial reaction. Because the infiltrating time is not only an important preparation process parameter but also one of the main factors affecting the interfacial reaction, it is the main aim of this work to study its influence on the interfacial reaction and the mechanical properties of the composite materials.

In this study, the tungsten particles reinforced Zr-based amorphous composites with different infiltrating times were prepared by the infiltrating casting method. The effects of infiltrating time on the interfacial reaction and crystal formation in the amorphous composites were investigated. Through the compression test of the amorphous composites at room temperature, the effects of infiltrating time on the compressive properties, fracture morphology and shear bands multiplication of the amorphous composites were explored.

\section{Experimental}

The ingots of $\mathrm{Zr}$ and Ti were produced by vacuum arc melting of sponge $\mathrm{Zr}$ and Ti blocks with a purity of $99.9 \%$ in a Tigettered argon atmosphere, respectively. The master ingots with the nominal composition of $\mathrm{Zr}_{41.2} \mathrm{Ti}_{13.8} \mathrm{Cu}_{12.5} \mathrm{Ni}_{10} \mathrm{Be}_{22.5}$ (Vit 1 alloy) were prepared by induction melting of a mixture of $\mathrm{Zr}, \mathrm{Ti}, \mathrm{Ni}, \mathrm{Cu}$ and Be componential materials with a purity of $99.9 \%$ or more in a quartz crucible in an argon atmosphere.
Tungsten particles with a size of 700-900 $\mu \mathrm{m}$ were cleaned with alcohol by an ultrasonic washer to keep surfaces clean and fresh, and then, were packed and sealed in the end of a U-shape quartz tube. The height of the packed tungsten particles was $70 \mathrm{~mm}$ in the quartz tube, then the particles were vacuumdried at $300{ }^{\circ} \mathrm{C}$ for $20 \mathrm{~min}$. Subsequently, they were heated to $780{ }^{\circ} \mathrm{C}$, about $40{ }^{\circ} \mathrm{C}$ higher than the melting point $\left(742{ }^{\circ} \mathrm{C}\right)$ of the master alloy of Vit $1 \mathrm{BMG}$, in the lower chamber of the electric resistance furnace which is divided into two chambers and controlled by respective temperature adjusters. At the same time, the charges of the prealloyed Vit 1 material in the U-tube were melted at $850{ }^{\circ} \mathrm{C}$ under high vacuum $\left(2.0 \times 10^{-3} \mathrm{~Pa}\right)$ in the upper chamber of the furnace. After $15 \mathrm{~min}$, the alloy was melted and formed a gas-tight seal against the wall of the tube, then the high-purity argon under the net pressure of $250 \mathrm{kPa}$ was admitted to the tube, forcing the molten melt to move firstly upwards then downwards and finally infiltrate into the tungsten particles. The infiltrating time was measured by a stopwatch, starting from the moment of argon flushing to the moment of sample quenching. The infiltrating time was controlled to be 1 , 5 and $10 \mathrm{~min}$, respectively. The rapid solidification of samples was realized by quenching the U-tube into a saturated brine. The BMGCs having a diameter of $6 \mathrm{~mm}$ and a length of $70 \mathrm{~mm}$ were thus obtained.

Compressive samples with the size of $\Phi 6 \mathrm{~mm} \times 12 \mathrm{~mm}$ were cut from the as-cast samples, and compressive tests were performed with a universal mechanical testing machine under the strain rate of $1 \times 10^{-4} \mathrm{~s}^{-1}$. Two compressive samples were tested for each separate infiltrating time, and the performance data were averaged from these two samples. The microstructures, phase constituents, fracture surfaces and shear bands of the samples were examined with the S-3400N scanning electron microscope (SEM).

\section{Results}

Figure 1 shows the morphology of the as-cast tungsten particles reinforced $\mathrm{Zr}$-based composites prepared by the liquid infiltrating method with different infiltrating times. It is observed that the monolithic amorphous structure is formed, and the tungsten particles are independently distributed in the composites. Since the matrix of the amorphous alloy is not separated by the tungsten particles, the compressive strength of the composite materials can be maintained.
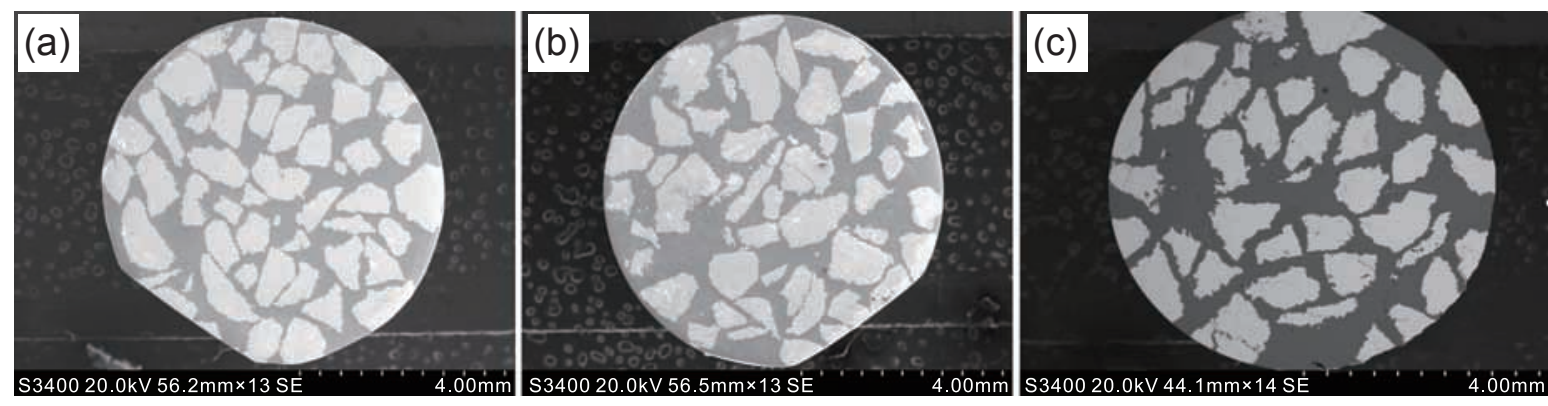

Fig. 1: Morphologies of tungsten particles reinforced Zr-based bulk metallic glass composites with different infiltrating times: (a) $1 \mathrm{~min}$; (b) $5 \mathrm{~min}$; (c) $10 \mathrm{~min}$ 
Figure 2 shows the interface morphology of the Zr-based amorphous composites reinforced by the tungsten particles with different infiltrating times. Figures 2(a) and (b) display the interface morphology of the sample with the infiltrating time of 1 min under different microscopic magnifications. It can be observed that some tiny nanocrystals are generated at the boundaries between the tungsten particles and the matrix, and micro-cracks appear in the tungsten particles, as shown by the arrows in Fig. 2(a). Figures 2(c) and (d) show the interface morphology of the sample with the infiltrating time of 5 min under different microscopic magnifications. It can be seen that no large crystals are generated in the matrix of the composite, but the solubility of the tungsten particles in the matrix increases. Furthermore, it can be observed that some of the white tungsten particles are cracked and split by the cracks. Therefore, it can be inferred that the interfacial reaction between the tungsten particles and the matrix becomes more obvious with the increase of infiltrating time. It can also be seen that many nanocrystals are generated at the boundaries, and some tiny dark crystals, as indicated by the arrows in Fig. 2(d), are found in the matrix near the boundaries. Figures 2(e) and (f) show the interface morphology of the sample with the infiltrating time of $10 \mathrm{~min}$. It can be obviously seen that the polygonal crystals appear in the matrix of the composite material, and the interfacial reaction at the boundaries between the tungsten particles and the amorphous matrix is apparently severe. It can also be seen that the micron-sized crystals are generated at the boundaries. This indicates that the degree of interfacial reaction in the composite materials increases with the infiltrating time. Due to the interfacial reaction, tiny nanocrystals are generated at the boundaries of the tungsten particles and the amorphous matrix, and furthermore, the size of the crystals increases with the infiltrating time. When the infiltrating time is $10 \mathrm{~min}$, the polygonal crystals are produced in the matrix of the composite, the size of which is increased from nanometer level to micrometer level. Moreover, as the infiltrating time increases, more microcracks are observed in the tungsten particles.
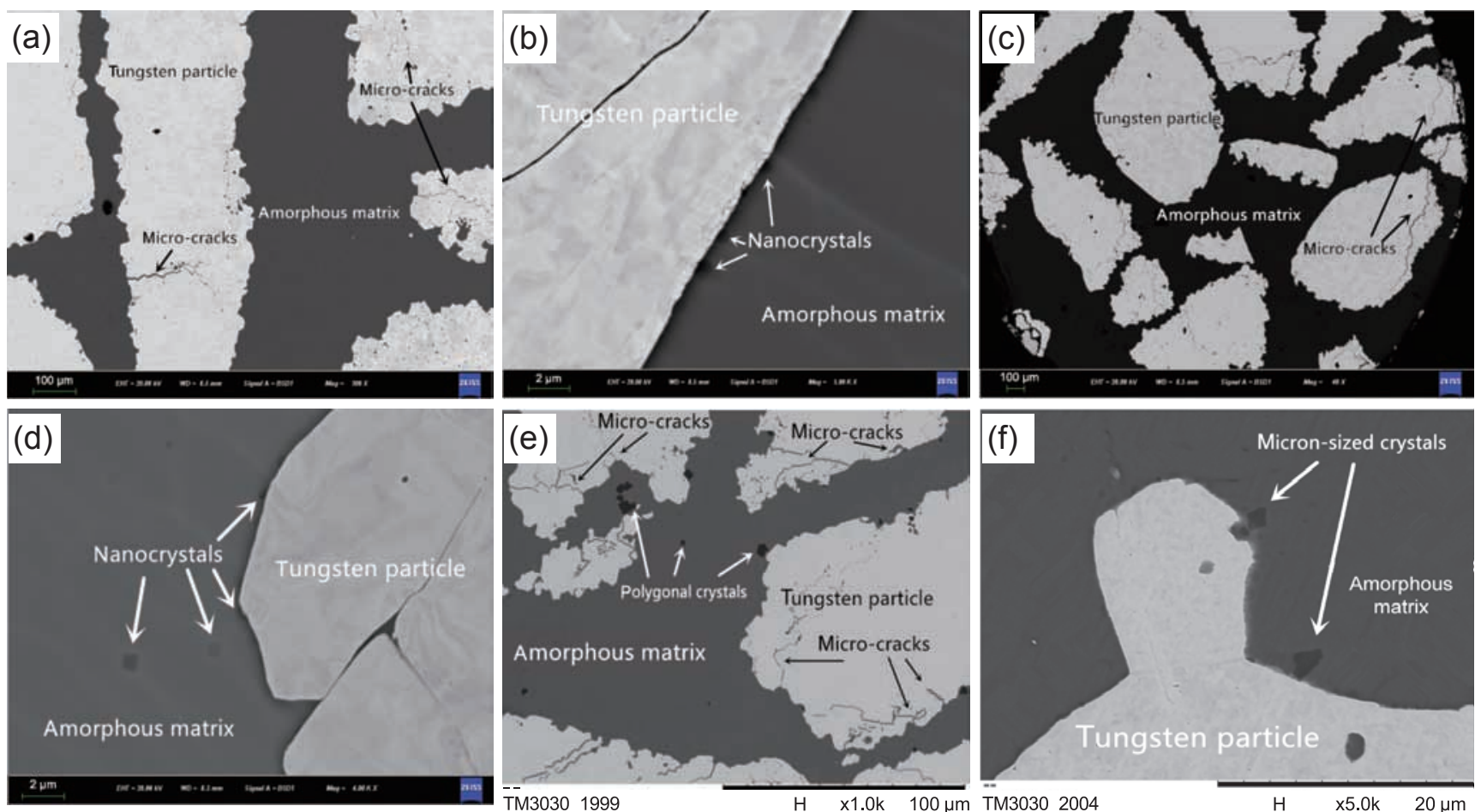

Fig. 2: Morphologies of interfacial reaction in tungsten particles reinforced Zr-based bulk metallic glass composites with different infiltrating times: (a and b) $1 \mathrm{~min}$; (c and d) $5 \mathrm{~min}$; (e and f) $10 \mathrm{~min}$

In order to explore the composition of the crystalline phases at the interfaces, the energy dispersive spectrum (EDS) tests on the generated crystals were conducted, and the EDS results are shown in Fig. 3. As shown in Figs. 3(a) and (b), Region A refers to the crystal generated at the interface and Region $B$ refers to the crystal generated in the matrix. Figure 3(c) is the EDS result of Region A. It is seen that the crystal generated at the interface mainly contains $\mathrm{W}, \mathrm{Ti}, \mathrm{Zr}$, and a small amount of $\mathrm{Cu}$ and Ni. Figure 3(d) is the EDS result of Region B, which shows that the crystal generated in the matrix mainly contains $\mathrm{Zr}$ and $\mathrm{Ti}$ elements, as well as a small amount of $\mathrm{Cu}$ and $\mathrm{Ni}$. This indicates that $\mathrm{W}$ element does not diffuse into the interior of the matrix during the preparation process, but participates in the interfacial reaction with the elements in the matrix at the interfaces to form tiny crystals growing therein. According to References [16-19], it is contemplated that with the extension of the infiltrating time, the interfacial reaction products of $\mathrm{W}_{5} \mathrm{Zr}_{3}$ and $\mathrm{CuTi}_{3}$ gradually appear near the interfaces, and within the matrix, the crystallization reaction is induced to generate the intermetallic compounds of $\mathrm{Zr}_{2} \mathrm{Cu}$ and $\mathrm{CuTi}_{3}$.

Figure 4 shows the compressive stress-strain curves of the three tungsten particles reinforced $\mathrm{Zr}$-based amorphous composite materials with different infiltrating times during the compression tests at room temperature. The compression rate used in the experiments was $1 \times 10^{-4} \mathrm{~s}^{-1}$. According to the compressive stress-strain curves, it can be interpreted that 

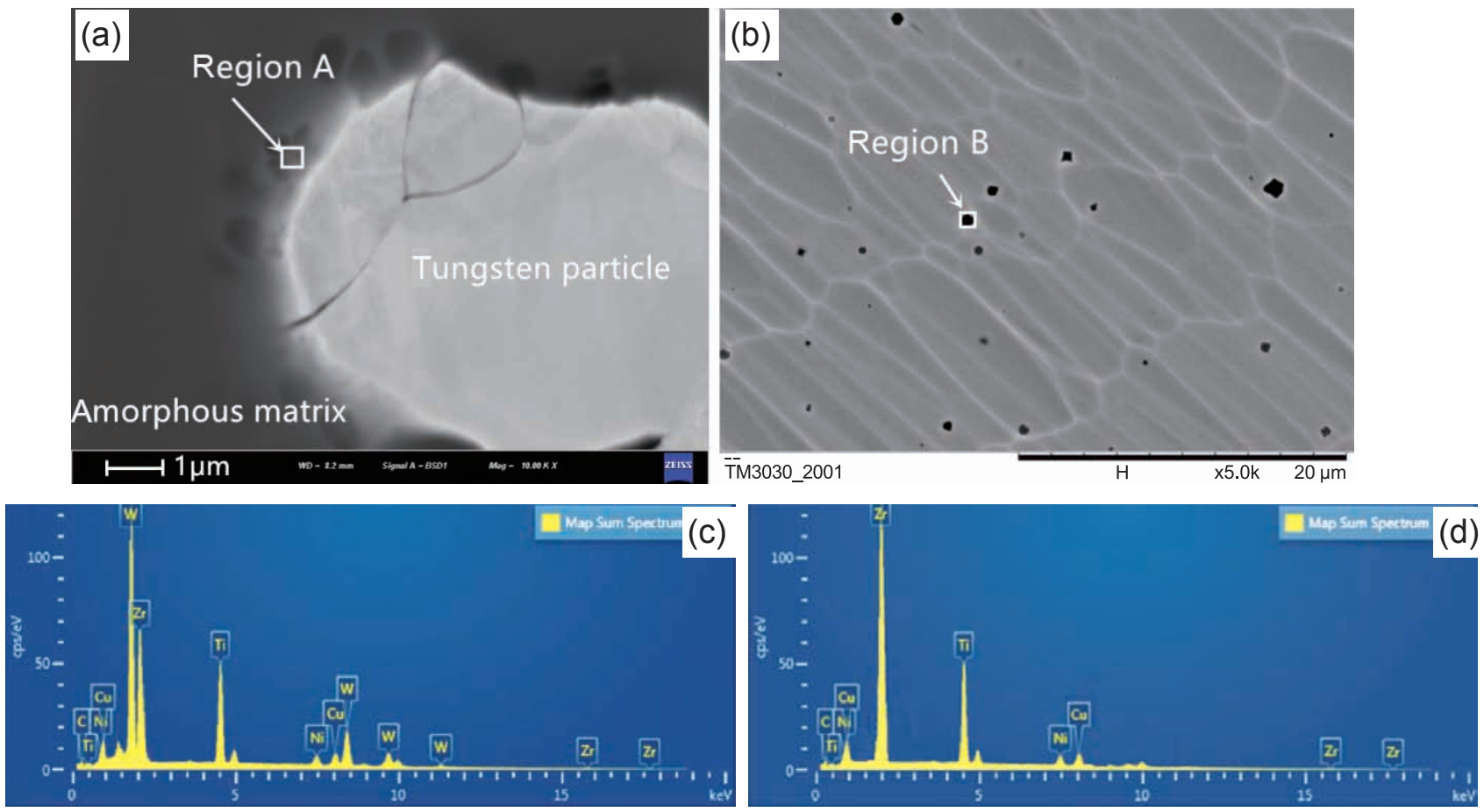

Fig. 3: EDS results of the crystals generated in tungsten particles reinforced Zr-based BMGs: (a) Region A; (b) Region B; and the energy spectrum analysis results: (c) Region A; (d) Region B

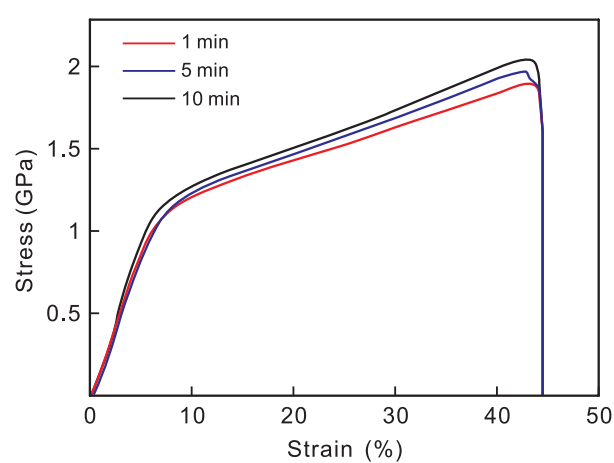

Fig. 4: Compressive stress-strain curves of tungsten particles reinforced $\mathrm{Zr}$-based bulk metallic glass composites with different infiltrating time

when the infiltrating time is $1 \mathrm{~min}$, the compressive strength of the composite material is $1,850 \mathrm{MPa}$, and the compressive strain is about $44 \%$, which is much better than the compressive plasticity ( $9 \%$ ) of the tungsten particles reinforced amorphous composites in Ref. [20]. As the infiltrating time is increased to $5 \mathrm{~min}$, the compressive strength of the composite material is increased to $1,960 \mathrm{MPa}$, but the compression strain does not change. When the infiltrating time is further increased to $10 \mathrm{~min}$, the compressive strength of the composite material reaches up to 2,030 $\mathrm{MPa}$, however, the compression strain still remains unchanged. In addition, it can be seen that all the three groups of composite materials begin to undergo plastic deformation when the compressive stress rises to about $1 \mathrm{GPa}$. It can be inferred from Fig. 4 that the compressive strength of the composite materials increases with the infiltrating time, but the infiltrating time has no effect on the compression strain of these materials.

Figure 5 shows the fracture morphology of the tungsten particles reinforced $\mathrm{Zr}$-based amorphous composites with different infiltrating times. Under room temperature compression, the fracture of the samples proceeds at the maximum shear stress direction (i.e., at an angle of approximately $45^{\circ}$ to the compressive loading direction), similarly as in the typical amorphous alloys ${ }^{[21,22]}$. It can be seen that the fracture surfaces are relatively flat, the fracture morphology of the matrix is a typically vein-shaped fracture, and there are many obvious melting phenomena ${ }^{[20,23]}$ observed on the fracture surfaces. Two types of veins are found in Figs. 5(b), (d) and (f), namely, the large size veins and the small size ones. The large size veins are presented in the amorphous matrix, while the small size ones are mostly concentrated near the boundaries between the tungsten particles and the amorphous matrix.

Figure 6 shows the morphology of the lateral shear bands in the tungsten particles reinforced $\mathrm{Zr}$-based amorphous composites with different infiltrating times. It is found in the figure that there are many micro-cracks and shear bands in the fractured composite materials. It can be interpreted that the deformation mode of the tungsten particles is mainly intergranular fracture, and the deformation mode of the amorphous matrix is the propagation of shear bands. It is seen that the shear bands in the amorphous composite materials are firstly formed at the boundaries between the tungsten particles and the amorphous matrix, and then propagate in the matrix phase at a specific direction, which is demonstrated by the arrows in the figure. Furthermore, during the propagation process, secondary shear bands are generated. The propagation of the shear bands only appears in the amorphous matrix, and is disrupted by the tungsten particles, indicating that the tungsten particles will hinder and inhibit the propagation of the shear bands. In addition, it is observed that as the infiltrating time increases, the density of the shear bands also increases. 


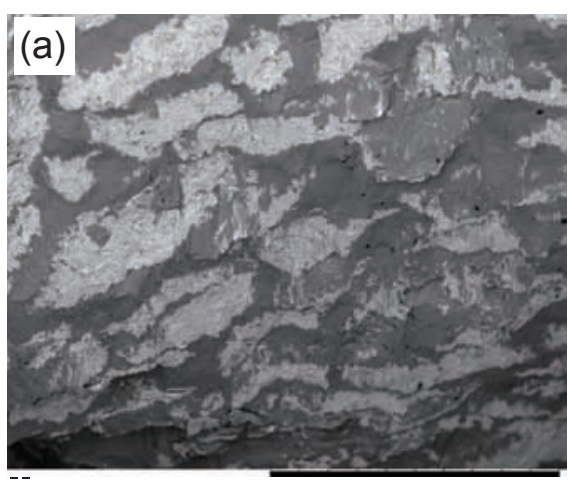

T̄ंM3030 1958

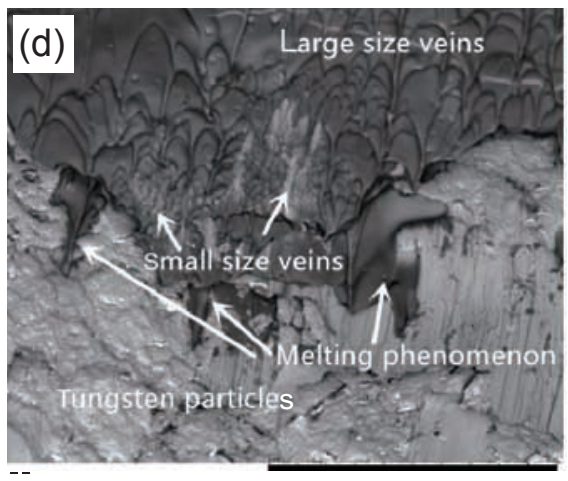

TM3030_1973

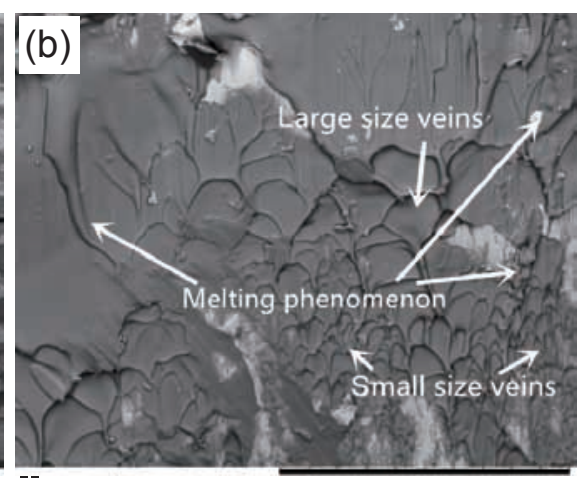

$\mathrm{H} \quad \mathrm{x} 1.0 \mathrm{k} \quad 100 \mu \mathrm{m}$ TM3030 1971

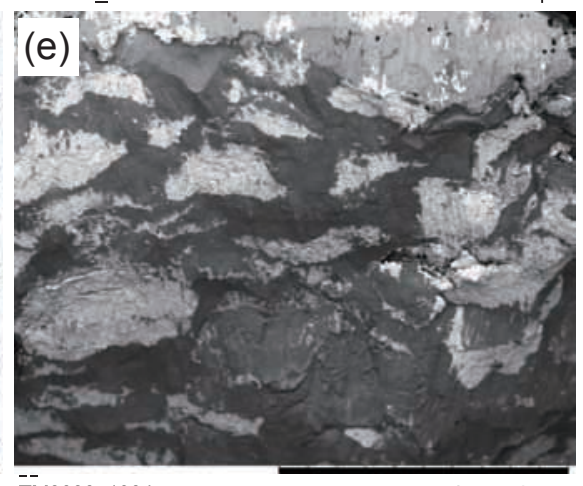

TM3030_1984

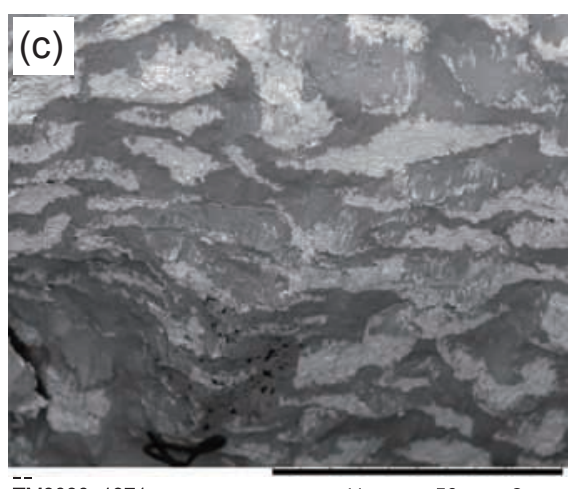

$\mathrm{H} \quad \mathrm{x} 50 \quad 2 \mathrm{~mm}$

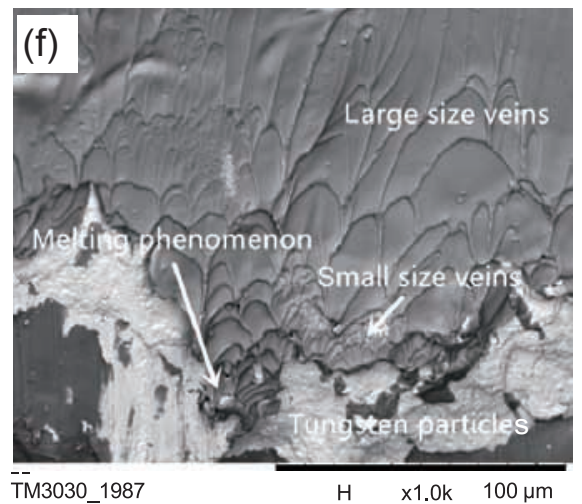

Fig. 5: Fracture morphologies of tungsten particles reinforced Zr-based amorphous composites with different infiltrating times: ( $a$ and b) $1 \mathrm{~min}$; (c and d) $5 \mathrm{~min}$; (e and f) $10 \mathrm{~min}$
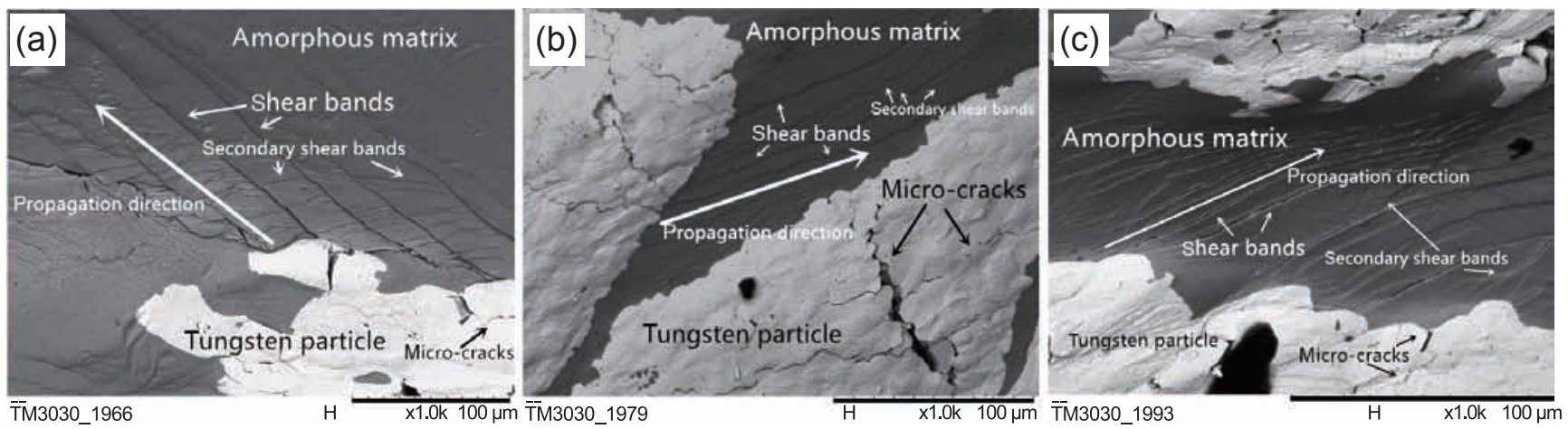

Fig. 6: Morphologies of lateral shear bands in tungsten particles reinforced Zr-based bulk metallic glass composites with different infiltrating times: (a) $1 \mathrm{~min}$; (b) $5 \mathrm{~min}$; (c) $10 \mathrm{~min}$

\section{Discussion}

Since the Zr-based alloy melt is reactive wetting to the tungsten particles, the time of the interfacial reaction, which is directly related to the infiltrating time, is an important factor affecting the performance of the composite materials.

The crystalline tungsten phases are not found in the amorphous matrix (Fig. 2) because of the high thermal stability of tungsten particles. The melting temperature of tungsten particles is $3,370{ }^{\circ} \mathrm{C}$, much higher than the maximum processing temperature $\left(850{ }^{\circ} \mathrm{C}\right)$. However, the diffusion of tungsten element is one of the mechanisms causing the interfacial reaction. The boundaries between the tungsten particles and the amorphous matrix are prone to facilitating heterogeneous nucleation to form crystals through the interfacial reaction. According to the reactive wetting mechanism, with increasing the time of interfacial reaction, the surface tension of the melt decreases continuously, causing reaction products to be easily transferred through the melt by the liquid convection. These reaction products can act as crystalline nuclei in the matrix, so that they would deteriorate the amorphous forming ability of the matrix ${ }^{[16]}$ and promote the formation of crystals within the amorphous matrix.

The compressive strength of the amorphous composite material increases with the increase of the infiltrating time. Since the tungsten particles and the amorphous matrix are metallurgically combined due to the interfacial reaction, the interfacial strength is one of the factors limiting the compressive strength of the composite materials. The interfacial reaction is conducive to the improvement of the interfacial strength, and the tiny crystals generated at the boundaries can produce the pinning effect at the interfaces ${ }^{[24]}$. As the infiltrating time increases, the number 
of crystals at the boundaries also increases. Therefore, although more micro-cracks are observed in the tungsten particles when the infiltrating time is $10 \mathrm{~min}$, the compressive strength of this composite material is still improved. In addition, the small number of tiny crystals within the amorphous matrix can be used as the in-situ reinforcing phase to affect the propagation of the shear bands, thereby enhancing the compressive strength of the amorphous composites. Thus, as the infiltrating time increases, the degree of interfacial reaction increases and the compressive strength of the composite materials also increases. It should be noticed that with excessive increase in the infiltrating time, as observed in the tungsten fiber reinforced $\mathrm{Zr}_{55} \mathrm{Al}_{10} \mathrm{Ni}_{5} \mathrm{Cu}_{30}$ BMGCs, severe interfacial reactions would occur and result in the decrease of fracture strength of the resultant composites ${ }^{[25]}$.

The fracture morphology of the composite materials is in a vein pattern, which is the classic morphology of the shear fracture of amorphous alloys. It is observed from Fig. 5 that because of the proximity to the boundaries between the tungsten particles and the amorphous matrix, the vein patterns tend to decrease in size. It is known that in amorphous alloys, the fracture surfaces generally show the vein patterns, as shear band planes are locally melted by the heat or free volume generated during deformation ${ }^{[20]}$. Large vein patterns imply the fast propagation speed of shear bands, as a considerable amount of plastic flow exists prior to local melting and solidification, whereas small vein patterns indicate the slow propagation speed of shear bands ${ }^{[26]}$. The decrease in the size of vein patterns near the boundaries indicates that the propagation of the shear bands in the amorphous matrix is interrupted by the tungsten particles, which confirms the role of tungsten particles in reinforcing the amorphous composite materials. A melting phenomenon is often observed on the compressive fracture surfaces of amorphous composites. This is caused by the strain energy of the sample during compression at two aspects: on the one hand, the sample failure increases the surface energy, on the other hand, the free volume and temperature of the shear bands are increased. When the strain energy accumulated is higher than the melting energy of pure amorphous alloy, it can cause the amorphous matrix at the local places of stress concentration to produce sufficiently high temperature or free volume ${ }^{[27]}$, and thus to be melted, especially near the tungsten particles.

On the stress-strain curves, the large range of complete plastic deformation of the composite materials reflects the formation process of multiple shear bands. It is reported that the elasticcomplete plastic behavior is common for amorphous composites when the aspect ratio of amorphous specimens is less than 1.5 , the low aspect ratio makes the compressed specimen in a plane strain state and limits the propagation of shear cracks ${ }^{[28]}$. According to Refs. [3, 4], the formation of multiple shear bands was found during the deformation process of the amorphous composites. These shear bands can be localized or uniformly distributed. When the initial displacement is very small, the shear bands are uniform. When the volume fraction of tungsten particles is large, the matrix is constrained by the tungsten particles, so the sliding displacement is low, and stress concentration is produced at the tip of the shear bands during propagation, which induces the formation of multiple shear bands, especially when the shear bands encounter the obstacles. In this study, with the increase of infiltrating time, the number of tiny crystals generated in the composite materials increases. Under the loaded compressive stress, the crystal phases can be served as the "nuclei" of the shear bands in the amorphous matrix, promoting the formation of the shear bands and increasing the number of multiple shear bands ${ }^{[29,30]}$. Therefore, with increasing the infiltrating time, during the compressive tests of the composite materials, the number of shear bands increases, and the gap width between the shear bands decreases, thus improving the compressive properties of the amorphous composite materials.

During the deformation process of the compressive samples, the ductile tungsten particles will yield under a relatively high yield stress and generate stress concentration, playing a major role in improving the mechanical properties of the composites. In addition, the ductile tungsten particles can hinder the propagation of shear bands and induce the formation of multiple shear bands, which further improve the compressive properties of the composites ${ }^{[20]}$. The tiny crystalline phase is generated at the interface due to the interfacial reaction, and with the increase of the infiltrating time, the polygonal crystals are generated in the amorphous matrix of the composite. According to Refs. [24, 31], the tiny nano-crystals and polygonal crystals in the composite materials exhibit brittleness. However, since both the number and size of the crystals are small, their brittleness does not affect the strength of the matrix. On the contrary, the tiny nano-crystals have a pinning effect on the interface which can improve the interface strength ${ }^{[24,19]}$, and the polygonal crystals can serve as an endogenous reinforcement phase in the composites. Both of them can also hinder the propagation of shear bands during the deformation process of the samples, thus improving the mechanical properties of the composite materials ${ }^{[18]}$.

The cause of micro-cracks on the tungsten particles is due to thermal stress. Since the melting point of the tungsten particles is $3,370^{\circ} \mathrm{C}^{[20,16]}$, the tungsten particles will hardly expand freely at the temperature of this experiment. During the melt infiltrating casting process of the composite, the temperature of tungsten particles is low, and the temperature of the molten melt is high. When they meet with each other, a temperature difference will occur, resulting in the formation of thermal stress and the microcracks of the tungsten particles in the composites ${ }^{[32,33]}$. As the infiltrating time increases, the thermal stress continues to act on the tungsten particles, so more micro-cracks are observed on the tungsten particles, as shown in Fig. 2. With the increase of micro-cracks on the tungsten particles, the amorphous melt is penetrated into the micro-cracks, and the number of interfaces between the tungsten particles and amorphous matrix is increased. Furthermore, it can be seen that the micro-cracks on the tungsten particles show an irregular shape, so the increased interfaces will cause a better effect of mechanical self-locking between the tungsten particles and the amorphous matrix, thus further improving the role of the tungsten particles in enhancing the mechanical properties of the composite materials. 


\section{Conclusions}

(1) Tiny crystals are prone to generate at the boundaries between the tungsten particles and amorphous matrix in the BMGCs, and the size of tiny crystals as well as the degree of interfacial reaction is increased with the infiltrating time.

(2) When the infiltrating time is $10 \mathrm{~min}$, the compressive strength of the composite reaches $2,030 \mathrm{MPa}$ and the compressive strain is $44 \%$. The increase of infiltrating time increases the compressive strength of the composites but has no significant effect on the compressive strain.

(3) During the compressive tests of the composite materials, with the increase of the infiltrating time, the number of shear bands increases, and the gap width between the shear bands decreases, thereby improving the compressive properties of the composite materials.

\section{Acknowledgement}

This work was supported by the Liaoning Joint Fund of NSFC (No. U1908219).

\section{References}

[1] Martin M, Thadhani N N. Mechanical properties of bulk metallic glasses. Progress in Materials Science, 2010, 55: 759-839.

[2] Hufnagel T C, Schuh C A, Falk M L. Deformation of metallic glasses: recent developments in theory, simulations, and experiments. Acta Materialia, 2016, 109: 375-393.

[3] Choi-Yim H, Johnson W L. Bulk metallic glass matrix composites. Applied Physics Letters, 1997, 71: 3808-3810.

[4] Choi-Yim H, Conner R D, Szuecs F, et al. Quasistatic and dynamic deformation of tungsten reinforced $\mathrm{Zr}_{57} \mathrm{Nb}_{5} \mathrm{Al}_{10} \mathrm{Cu}_{15.4} \mathrm{Ni}_{12.6}$ bulk metallic glass matrix. Scripta Materialia, 2001, 45: 1039-1045.

[5] Choi-Yim H, Schroers J, Johnson W L. Microstructures and mechanical properties of tungsten wire/particles reinforced $\mathrm{Zr}_{57} \mathrm{Nb}_{5} \mathrm{Al}_{10} \mathrm{Cu}_{15.4} \mathrm{Ni}_{12.6}$ metallic glass matrix composites. Applied Physics Letters, 2002, 80: 1906-1908.

[6] Martin M, Kecskes L, Thadhani N N. High-strain-rate dynamic mechanical behavior of a bulk metallic glass composite. Journal of Materials Research, 2008, 23: 998-1007.

[7] Zhang $X Q$, Wang $L$, Xue $Y F$, et al. Effect of the metallic glass volume fraction on the mechanical properties of Zr-based metallic glass reinforced with porous $W$ composite. Materials Science \& Engineering A, 2013, 561: 152-158.

[8] Zhang B, Fu H, Zhu Z, et al. Synthesis and properties of tungsten balls-Zr-base metallic glass composite. Materials Science and Engineering A, 2012, 540: 207-211.

[9] Pan J, Lin Y, Zhang J, et al. Effect of Ta particles on the fracture behavior of notched bulk metallic glass composites. Intermetallics, 2019, 106: 1-6.

[10] Xue Y F, Wang L, Cheng H W, et al. Dynamic tensile property of Zrbased metallic glass/porous $W$ phase composite. Journal of Materials Science \& Technology, 2010, 26: 908-913.

[11] Li H, Subhash G, Kecskes L J, et al. Mechanical behavior of tungsten preform reinforced bulk metallic glass composites. Materials Science \& Engineering A, 2005, 403: 134-143.

[12] Lee J C, Kim Y C, Ahn J P, et al. Enhanced plasticity in a bulk amorphous matrix composite-macroscopic and microscopic viewpoint studies. Acta Materialia, 2005, 53: 129-139.

[13] Ye C, Xue J X, Liu T, et al. Evolution of microstructure and nanohardness of SiC fiber-reinforced $\mathrm{SiC}$ matrix composites under Au ion irradiation. Ceramics International, 2020, 46(6): 8165-8173.
[14] Wang P, Yu B Q, Fan Q B, et al. Anisotropic dynamic mechanical response of tungsten fiber/Zr-based bulk metallic glass composites. Materials \& Design, 2016, 93: 485-493.

[15] Xue $Y F$, Zhong $X$, Wang $L$, et al. Effect of $W$ volume fraction on dynamic mechanical behaviors of $\mathrm{W}$ fiber/Zr-based bulk metallic glass composites. Materials Science \& Engineering A, 2015, 639: 417-424.

[16] Khademian N, Gholamipour R. Study on microstructure and fracture behavior of tungsten wire reinforced Cu-based and $\mathrm{Zr}$-based bulk metallic glass matrix composites. Journal of Non-Crystalline Solids, 2013, 365: 75-84.

[17] Xu Q G, Qiu K Q, et al. Wetting and composite between $\mathrm{Zr}_{41.25} \mathrm{Ti}_{13.75} \mathrm{Ni}_{10} \mathrm{Cu}_{12.5} \mathrm{Be}_{22.5}$ molten and W. Rare Metal Materials and Engineering, 2007(5): 813-816.

[18] Liu X F, Chen Y, Dai L H. Deformation field evolution and shear band behavior of endogenous crystal amorphous alloy composites. Science in China: Physics, Mechanics, Astronomy, 2020, 50(6): 4957.

[19] Ma W F, Kou H C, Li J H, et al. The present status of interface study of $\mathrm{W}$ fiber reinforced $\mathrm{Zr}$-based amorphous matrix composite. Materials Reports, 2006, 20(4): 64-66.

[20] Qiu K Q, Ren Y L. Tungsten particle reinforced bulk amorphous composite material. Rare Metal Materials and Engineering, 2006, 35: 66-69.

[21] Johnson W L. Bulk glass-forming metallic alloys: science and technology. Matrials Research Bulletin, 1999, 10: 42-56.

[22] Zhang Z F, Zhang H, Shen B L, et al. Shear fracture and fragmentation mechanisms of bulk metallic glasses. Philosophical Magazine Letters, 2006, 86: 643-650.

[23] Tabachnikova E D, Bengus V Z, Stolyarov V V, et al. The contribution of grain boundary dislocations to the plastic deformation of nanostructured titanium from the SD-effect of the yield stress. Materials Science \& Engineering A, 2001, 309-310: 524-527.

[24] Li W, Chen D, Chen Z H, et al. Effect of endogenic crystal phase on mechanical properties of $\mathrm{Cu}_{46} \mathrm{Zr}_{46} \mathrm{Al}_{8}$ bulk metallic-glass matrix composites. Transactions of Materials and Heat Treatment, 2010, 31(8): 1-5.

[25] Qiu K Q, Wang A M, Zhang H F, et al. Melt infiltration casting of $\mathrm{Zr}_{55} \mathrm{Al}_{10} \mathrm{Ni}_{5} \mathrm{Cu}_{30}$ bulk metallic glass matrix composite. Chinese Journal of Materials Research, 2000, 16(4): 389-394.

[26] Choi-Yim H, Busch R, Koster U, et al. Synthesis and characterization of particulate reinforced $\mathrm{Zr}_{57} \mathrm{Nb}_{5} \mathrm{Al}_{10} \mathrm{Cu}_{15.4} \mathrm{Ni}_{12.6}$ bulk metallic glass composites. Acta Materialia, 1999, 47(8): 2455-2462.

[27] Connner R D, Dandliker R B, Johnson W L. Mechanical properties of tungten and steel fiber reinforced $\mathrm{Zr}_{41.25} \mathrm{Ti}_{13.75} \mathrm{Cu}_{12.5} \mathrm{Ni}_{10} \mathrm{Be}_{22.5}$ metallic glass matrix composites. Acta Materialia, 1998, 46(17): 6089-6102.

[28] Qiu K Q, Wang A M, Zhang H F, et al. Tungsten wire reinforced ZrAINiCuSi bulk amorphous composites and their plastic behavior. Acta Metallurgica Sincia, 2002, 38(10): 1091-1096.

[29] Lai Z H, Conrad H, Teng G Q, et al. Nanocrystallization of amorphous Fe-Si-B alloys using high current density electropulsing. Materials Science \& Engineering A, 2000, 287: 238-247.

[30] Qu S B, Yao K F. Crystallization kinetics of $\mathrm{Zr}_{41} \mathrm{Ti}_{14} \mathrm{Cu}_{12.5} \mathrm{Ni}_{10} \mathrm{Be}_{22.5}$ bulk metallic glass in pulsing current pretreatment states. Journal of Alloys and Compounds, 2009, 475: L5-L8.

[31] Qiu S B, Yao K F, Gong P. Effect of crystallization fraction on mechanical properties of $\mathrm{Zr}$-based bulk amorphous composite materials. Science in China: Physics, Mechanics, Astronomy, 2010, 40(6): 711-716.

[32] Cai Q H, Zou Y Q. The effect of interface layer and thermal stress in fiber-reinforced ceramics on matrix cracking. Journal of Tsinghua University (Natural Science Edition), 1998 (12): 84-88.

[33] Fukuda M, Nogami S, Guan W H, et al. Analysis of the temperature and thermal stress in pure tungsten monoblock during heat loading and the influences of alloying and dispersion strengthening on these responses. Fusion Engineering and Design, 2016, 107: 4450 